STRUCTURAL BIOLOGY
Received 25 September 2020

Accepted 8 April 2021

Edited by M. Schiltz, Fonds National de la Recherche, Luxembourg

\# Current address: Astex Pharmaceuticals, 436 Cambridge Science Park, Milton Road, Cambridge CB4 OQA, United Kingdom.

Keywords: fragment screening; high-throughput data analysis; protein crystallography; drug discovery; fragment-based lead discovery.

Supporting information: this article has supporting information at journals.iucr.org/d

\section{FragMAXapp: crystallographic fragment-screening data-analysis and project-management system}

\author{
Gustavo M. A. Lima, ${ }^{a} \ddagger$ Elmir Jagudin, ${ }^{a}$ Vladimir O. Talibov, ${ }^{a}$ Laila S. Benz, ${ }^{b}$ \\ Costantino Marullo, ${ }^{a}$ Tatjana Barthel, ${ }^{c}$ Jan Wollenhaupt, ${ }^{c}$ Manfred S. Weiss ${ }^{\mathrm{c}}$ and \\ Uwe Mueller ${ }^{\mathrm{c} *}$
}

\begin{abstract}
aBioMAX, MAX IV Laboratory, Fotongatan 2, 22484 Lund, Sweden, ${ }^{\mathbf{b}}$ Institut für Chemie und Biochemie, Freie Universität Berlin, Thielallee 63, 14195 Berlin, Germany, and 'Macromolecular Crystallography, Helmholtz-Zentrum Berlin, Albert-Einstein-Strasse 15, 12489 Berlin, Germany. *Correspondence e-mail: uwe.mueller@helmholtz-berlin.de
\end{abstract}

Crystallographic fragment screening (CFS) has become one of the major techniques for screening compounds in the early stages of drug-discovery projects. Following the advances in automation and throughput at modern macromolecular crystallography beamlines, the bottleneck for CFS has shifted from collecting data to organizing and handling the analysis of such projects. The complexity that emerges from the use of multiple methods for processing and refinement and to search for ligands requires an equally sophisticated solution to summarize the output, allowing researchers to focus on the scientific questions instead of on software technicalities. FragMAXapp is the fragmentscreening project-management tool designed to handle CFS projects at MAX IV Laboratory. It benefits from the powerful computing infrastructure of largescale facilities and, as a web application, it is accessible from everywhere.

\section{Introduction}

Fragment-based lead discovery utilizes a toolbox of biophysical methods, with X-ray crystallography-based fragment screening (CFS) being the main screening technique to obtain $3 \mathrm{D}$ structural information on protein-ligand complexes. In its methodological development, CFS co-evolves together with the scientific instrumentation and, more importantly, with the scientific infrastructure that supports contemporary macromolecular X-ray crystallography (MX) (Davies \& Tickle, 2011; Grimes et al., 2018). In its current state, CFS relies on experimental setups and data-analysis methodologies that deviate from a classical crystallographic workflow (Krojer et al., 2017; Davies \& Tickle, 2011; Wollenhaupt et al., 2021).

Advances in MX data acquisition are setting new standards in structural biology (Grimes et al., 2018). With the advent of MX beamlines at fourth-generation photon sources at synchrotron-radiation facilities such as BioMAX at MAX IV Laboratory (Ursby et al., 2020), Manacá at the SIRIUS Light Source (Nascimento, 2020), AMX and FMX at NSLS II (Fuchs et al., 2016) and MASSIF-1, MASSIF-2 and MASSIF-3 at ESRF-EBS (von Stetten et al., 2020; McCarthy et al., 2018; Svensson et al., 2018), the average data-collection time has been reduced by a factor of ten to less than $40 \mathrm{~s}$ for a $360^{\circ} \omega$ scan. With improved beamline sample capacity allied with reliable and fast robotic sample handling (Martiel et al., 2020; Ursby et al., 2020; Murakami et al., 2020), automated crystal centring (Ito et al., 2019; Di Castro et al., 2008; Schurmann et al., 2019; Svensson et al., 2020) and the possibility of 
unattended data collections (Mueller et al., 2017; Cipriani et al., 2012; Nurizzo et al., 2016; Sanchez-Weatherby et al., 2019), contemporary MX experiments generate a massive volume of data within a short time frame, for example more than 100 data sets during a single $8 \mathrm{~h}$ session. Finally, advances in crystal-harvesting including new tools (Barthel et al.., 2021) as well as new methods such as acoustic crystal harvesting (Samara et al., 2018; Yin et al., 2014), robot-aided harvesting (Wright et al., 2021) and fully automated systems (Cipriani et al., 2012) are increasing the number of crystals harvested by an order of magnitude. Thus, collecting data for CFS at such facilities is a relatively robust procedure and the main bottleneck in MX-based fragment-screening experiments is shifting from sample preparation and data acquisition to data analysis, the building of multiple models and the interpretation of the results.

Currently, automated methods for data processing and structure determination are the best way to deal with the enormous amount of data created in screening experiments. Although those methods are becoming very efficient, one major limitation is still computing power. For example, using a reasonably performing desktop computer, indexing and integrating a single high-resolution data set collected using a highresolution detector such as an EIGER 16M can take up to $1 \mathrm{~h}$ $30 \mathrm{~min}$. Automated and parallel approaches for data analyses benefit from exploring the solutions from several pipelines and cherry-picking the best solution, but the problem of limited computing power is aggravated. To facilitate parallel data processing for hundreds of data sets, data-collection facilities are investing in high-performance computing (HPC). Facilities such as MAX IV provide user access to their computing infrastructure, with the as-yet underestimated advantages of local availability of the data. CFS can benefit greatly from HPC-mediated data processing and analysis, as it improves the quality of the screening results and may possibly increase the sensitivity of the method.

The massive amount of raw experimental data, its processing and the refinement of all potential structures of proteinligand complexes, the exploration of bound ligands and the corresponding ligand-binding sites, and meta analysis of the data must be treated integrally. This concept was pioneered and first implemented by Astex Therapeutics Ltd (Cambridge, UK; currently part of Astex Pharmaceuticals) within their Pyramid Platform (Davies et al., 2006; Davies \& Tickle, 2011). A similar logic was applied by XChem, a fragment-screening facility at Diamond Light Source, Didcot, UK that has operated since 2015. XChem users manage CFS data with a standalone graphical application called XChemExplorer (Krojer et al., 2017), which is also available within the CCP4 software suite (Winn et al., 2011). The application guides the user through most stages of the screening experiment, including data processing, analysis, refinement of the models and their deposition in the Protein Data Bank (Berman et al., 2000). Another implementation of the concept was made by EMBL with the web-based Crystallographic Information Management System (CRIMS). This application records all experiments performed with a given target and allows automated data analysis using Pipedream (Vonrhein et al., 2011; Smart et al., 2014; Bricogne et al., 2017; Kabsch, 2010; Joosten et al., 2011; Collaborative Computational Project, Number 4, 1994).

Here, we present FragMAXapp, our solution to facilitate the scientific analysis, presentation and storage of large data sets from CFS and similar high-throughput crystallographic experiments (https://maxiv.lu.se/fragmax/fragmaxapp). FragМАХарp is a web-based expert system that links all of the steps of crystallographic ligand screening from experimental design and sample preparation to the deposition of the final structures in the Protein Data Bank. The application focuses on user accessibility, flexibility of the data analysis and high performance, linking the MAX IV HPC infrastructure with a selection of automated data-processing and analysis workflows (Fig. 1a). FragMAXapp was designed within the operation framework of the BioMAX beamline (Ursby et al., 2020) and connects to other beamline applications, such as the ISPyB sample database (Beteva et al., 2006) and the $M X C u B E$ experiment-control software (Mueller et al., 2017; Oscarsson et al., 2019). However, the abstraction level of FragMAXapp provides modularity that allows it to be ported and adapted to other sites. In fact, at the time of this publication, working versions of FragMAXapp are deployed on BESSY II at the Helmholtz-Zentrum Berlin (Mueller et al., 2015; Wollenhaupt et al., 2021) and at the University of São Paulo using data from the SIRIUS Light Source (Noske et al., 2021).

\section{Software design and operation}

FragMAXapp has been developed using Python3/Django (Django version 2.2.1; https://jangoproject.com) as its back end and using JavaScript for front-end features (Fig. 1b). The project definition, including protein models, the structures of the small molecules and user information, is stored in an SQLite3 database.

As a web application, FragMAXapp requires a web browser with the support of WebGL (Web Graphics Library API, Mozilla). This feature allows data analysis to be performed using virtually any device connected to the internet. The user interface is designed using HTML5, CSS3 and JavaScript for responsiveness and functionality. User access can be obtained using the ISPyB authentication system, which provides access to data and results based on the MAX IV user-account system, or with a local account with access privileges granted by the system administrator.

The application offers a variety of choices for data processing, including DIALS (Winter et al., 2018) and XDS (Kabsch, 2010) through xia2 (Winter et al., 2018; Evans, 2006; Gildea et al., 2011), autoPROC (Vonrhein et al., 2011), XDSAPP (Krug et al., 2012; Sparta et al., 2016), fastdp (Winter \& McAuley, 2011) and EDNAproc (Incardona et al., 2009). Automated structure refinement is performed by DIMPLE, BUSTER (Bricogne et al., 2017; Smart et al., 2012) and fspipeline (Schiebel et al., 2016). The ligand-finding step is performed by Rhofit (Smart et al., 2014), Phenix LigandFit (Terwilliger et al., 2006, 2007) and PanDDA (Pearce et al., 2017), with ligand coordinates and restraints generated by 
Phenix eLBOW (Moriarty et al., 2009), GRADE (Smart et al., 2011) or AceDRG (Long et al., 2017). Within the application interface, structural representation of the ligands is created using the RDKit (Landrum, 2012) and 3Dmol (Rego \& Koes, 2015) libraries. Protein atomic coordinates, electron-density maps and reciprocal space are displayed using the UglyMol (Wojdyr, 2016) library with minor modifications that allow the visualization and comparison of multiple models. Interactive plots for data statistics are created using the D3 library (Bostock, 2017). The X-ray diffraction representations are created using $A d x v$ (Arvai, 2012) with sufficient images to cover $1^{\circ}$ of crystal oscillation. The selection of software available in a specific facility is defined during the deployment of the application.

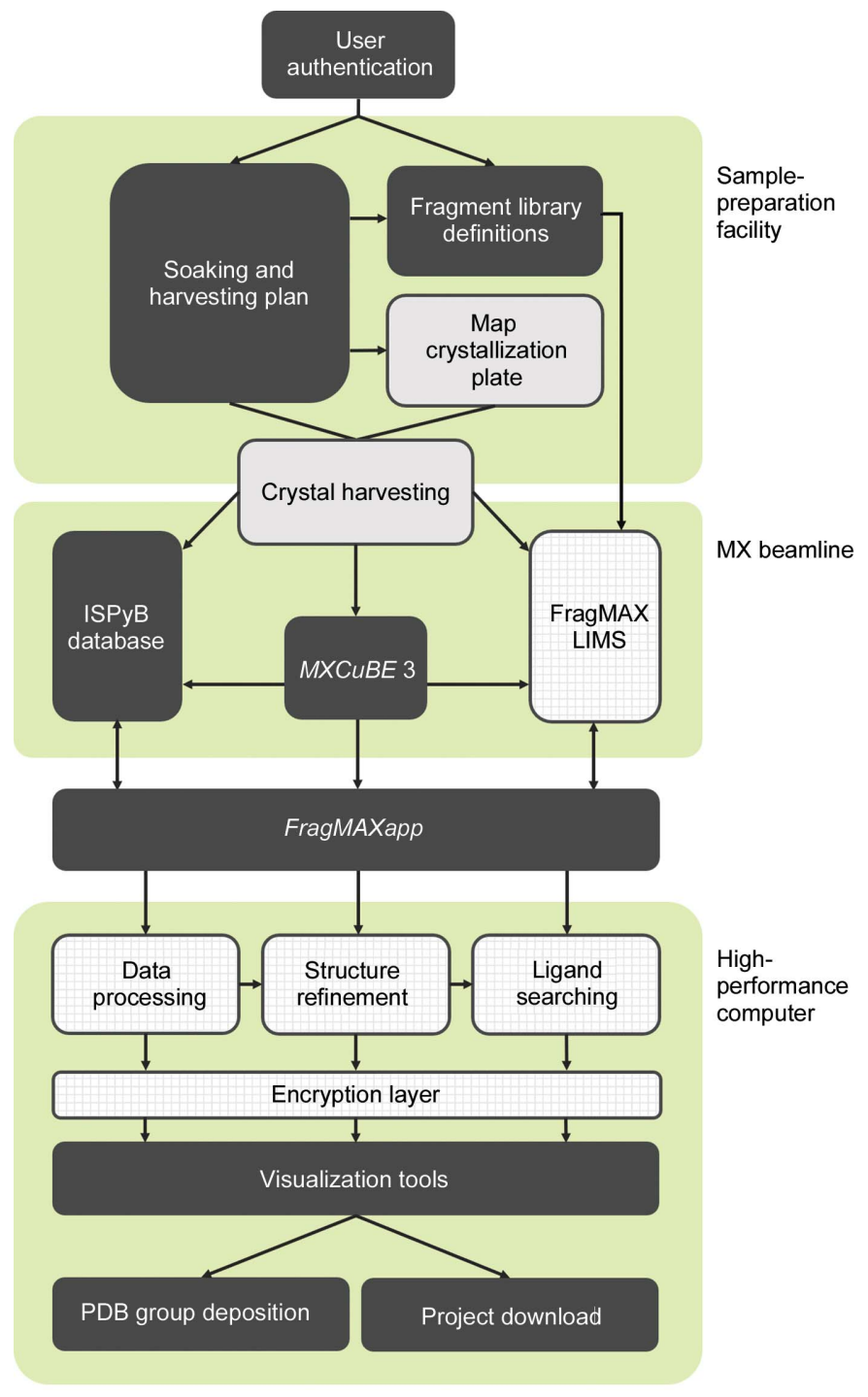

Figure 1

(a)
The MAX IV HPC infrastructure manages its workload using Slurm, which is interfaced with the FragMAXapp server using a Celery workload manager and a Redis server. These two services run together with the Django server to enable promise-like requests for data while maintaining fluid navigation in the web app.

All services run from a Docker container allocated in a virtual machine configured with GitHub's continuous integration feature, allowing easy deployment and updating of the tools. The Docker deployment uses NGINX/uWSGI to serve the application.

\subsection{Project management}

The application organizes experimental data and their analysis into application-specific projects. A FragMAXapp project is defined by a unique combination of four parameters: the protein name, a common identifier of the compounds (for example the name of the fragment library), the BioMAX proposal number and the corresponding data-collection sessions (Supplementary Fig. S1). These values can be updated to incorporate follow-up experiments related to the same protein within the same proposal.

To facilitate work with sensitive information and to allow users to follow up specific policies for data handling, the project can be created in an encrypted mode. With this option the project-related data are encrypted, and are decrypted only temporarily when the user or the processing software require access. For increased security, the user can manage the
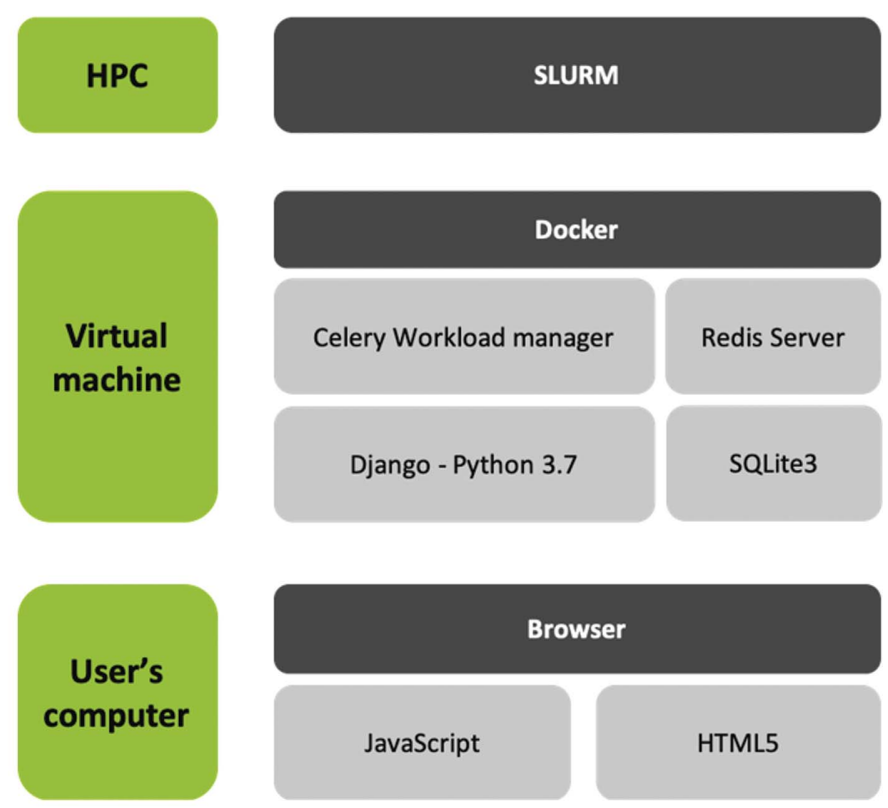

(b)

(a) FragMAXapp schematic diagram. The diagram shows connected steps and the relationship between each interaction, process and database entry. It is divided into three groups based on where the actions take place. Steps coloured dark grey use the HTML interface for interaction, light grey steps are tasks performed in the experimental laboratory and chequered steps are tasks performed in the background (for example HPC jobs). (b) FragMAXapp development design. Processing jobs are controlled by a Slurm workload manager installed in the front end of the MAX IV HPC. The three servers and the database necessary for FragMAXapp are deployed inside a Docker container inside a virtual machine, ensuring performance and stability of the installation. The user interface is designed using HTML5, CSS3 and JavaScript, which are available in all modern browsers. 
availability of the encryption key. It is possible to download, remove and re-upload the encryption key that is used for the project. When the encryption key is removed, the project data effectively become inaccessible unless the user re-uploads the key.

Beyond meta-management of the projects, FragMAXapp allows the examination of individual data sets and other experimental details. Often, this information is stored in sample databases such as ISPyB and can be retrieved at any time by the user. With FragMAXapp, browsing this information is also possible (Fig. 2).

\subsection{Sample management}

Definition of the screening collection is an essential step in project management. FragMAXapp requires the user to upload a comma-separated file (CSV) with library specifications, including compound identifiers, which must be identical to the sample names, and optional SMILES strings for the chemical structures of the ligands. The library view page displays updated information about the sample database, with 2D and/or 3D representations of each molecule, and the SMILES code for each compound ID in the project (Fig. 3). Missing entries in the sample database are displayed in the page header, based on the sample names available and the compound IDs in the database. It is possible to update the definition of the samples, which allows work with follow-up experiments within the same project.

\subsection{Data processing and structure solution}

The most powerful feature of FragMAXapp is its capability to handle numerous data-analysis methods in a parallel and multiplexed manner (Fig. 4). It is widely accepted that no single combination of processing and analysis software outperforms any available solution, meaning that only after testing all possible combinations of automated pipelines is it possible to identify the best strategy for a particular data set (Powell, 2017). As we focus on fully automated data-analysis schemes, FragMAXapp offers a combination of six dataprocessing software packages: autoPROC, xia2/DIALS, $x i a 2 / X D S$, EDNAproc, fastdp and XDSAPP, three structurerefinement pipelines, BUSTER, DIMPLE and fspipeline, and three automatic ligand-searching programs, Phenix LigandFit, Rhofit and PanDDA. Selecting all available software can generate a total of 54 combinations for the evaluation of each diffraction data set. For each software, FragMAXapp suggests options to optimize its function, such as the definition of resolution cutoffs for the data, alternative frame ranges for the data sets, the application of Friedel's law during processing, the water-placement method in structure-refinement stages and many more, efficiently acting as a unified GUI front end. Providing customized parameters for data analyses is optionally possible; otherwise the application chooses default values when no input is given. Using a built-in selection table, only selected data sets can be analysed or re-analysed. To ensure that all software will have the necessary information to run properly, FragMAXapp keeps a set of mandatory inputs.

\section{Data Collection Overview}

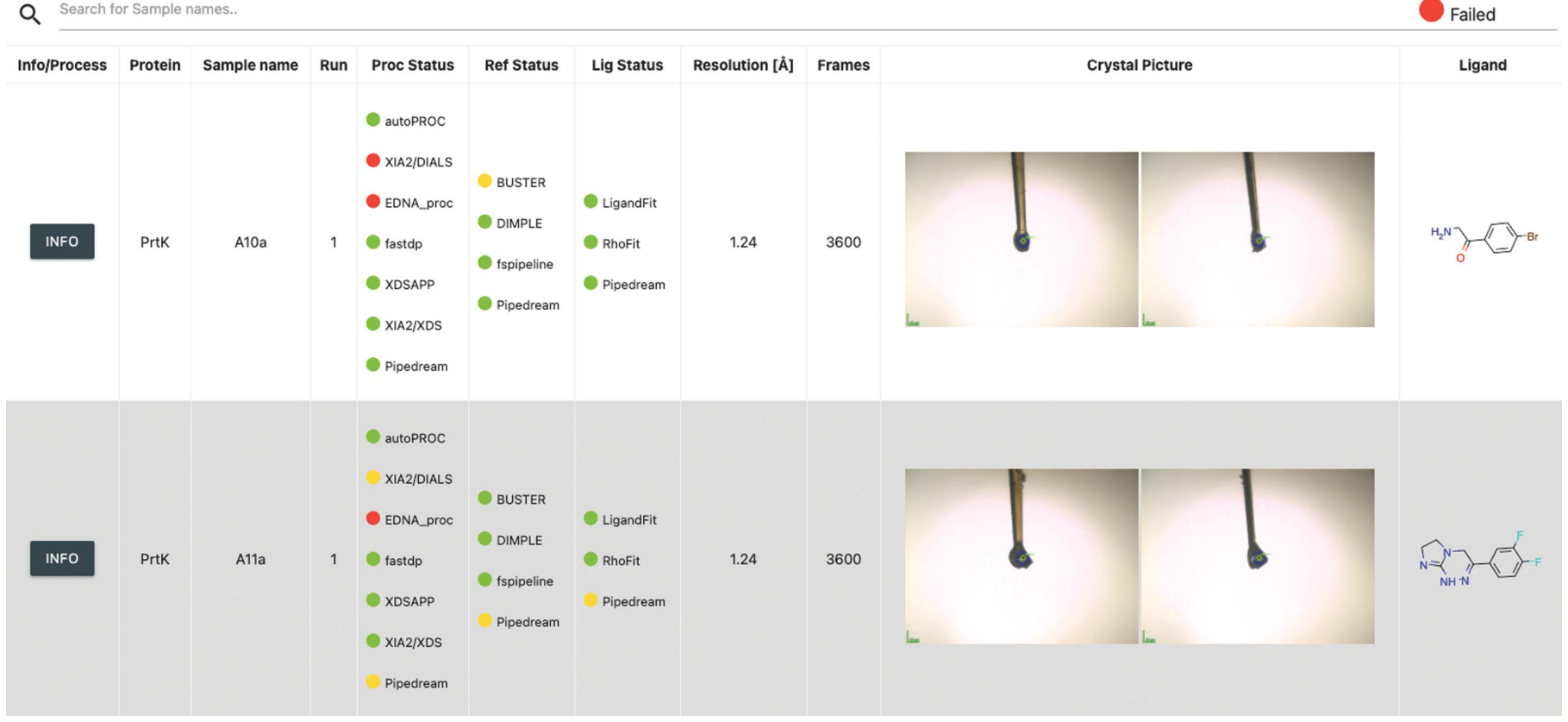

Figure 2

FragMAXapp Project Overview page. It displays information about the data collection and the status of data processing. A full representation of the user interface is available in Supplementary Fig. S2. 
A dedicated tab is available to check all currently running data-analysis jobs and inspect the processing logs. This feature displays live information from the computing nodes and the queueing system. Within this page, it is possible to remove jobs from the processing queue or to kill running analyses.

FragMAXapp integrates PanDDA analysis, with several features that allow the sensitivity of the screening experiment to be increased. Under the PanDDA tab on the Data Analysis page, it is possible to choose which data-analysis output will be used to run PanDDA. If the result is missing, FragMAXapp will check whether a solution was offered by other combinations of analysis software. Alternatively, the application offers the FragPLEX option, a method to decide which results to submit for PanDDA analysis. The method is based on the evaluation of processing and refinement statistics. The first step of the selection process discards all processed data sets with an overall $R_{\text {meas }}$ of $>10 \%$. The second step ranks solutions by higher ISa value and resolution and lower $R_{\text {free }} / R_{\text {work }}$. The selection is saved in the FragMAXapp database. In our tests, applying this selection logic allows the retrieval of all hits found by combining hits from all individual analysis (i.e. a single combination of data processing and structure refinement). Additionally, FragMAXapp offers the option to build ground-state models using exclusively known apo structures or to use data sets without peaks in the $Z$-maps (the latter requires at least one PanDDA analysis before use). In the final steps of $P a n D D A$ analysis, the evaluation of modelled ligands can be performed through the PanDDA Giant scoring scripts (Pearce et al., 2017), and the radar plots of fitting quality generated in this step are shown in the web app.

\subsection{Data visualization}

The results generated by the automatic analysis pipelines can be visualized in the web browser without any external tools or plugins. It is possible to open and read the output logs from each software used, visualize reciprocallattice representation and load refined models with electron density with a Coot-like look and feel using UglyMol (Fig. 5). Besides, the web app provides interactive and comprehensive overview plots for many statistical parameters such as resolution, ISa, $R_{\text {work }} / R_{\text {free }}$ and unit-cell parameters. This allows the whole CFS data evaluation to be compared within one view, including averaged values and standard deviations for various combinations of methods. These plots can be extremely helpful to obtain a quick overview of the data analysis and to identify data sets that require reprocessing or manual examination.

The PanDDA results visualization within FragMAXapp displays the event map and the average map obtained from the ground-state model side by side (Supplementary Fig. S6). Using this visual feature, it is easier to interpret unexplained or modelled densities that are also present in the averaged ligandfree model. 


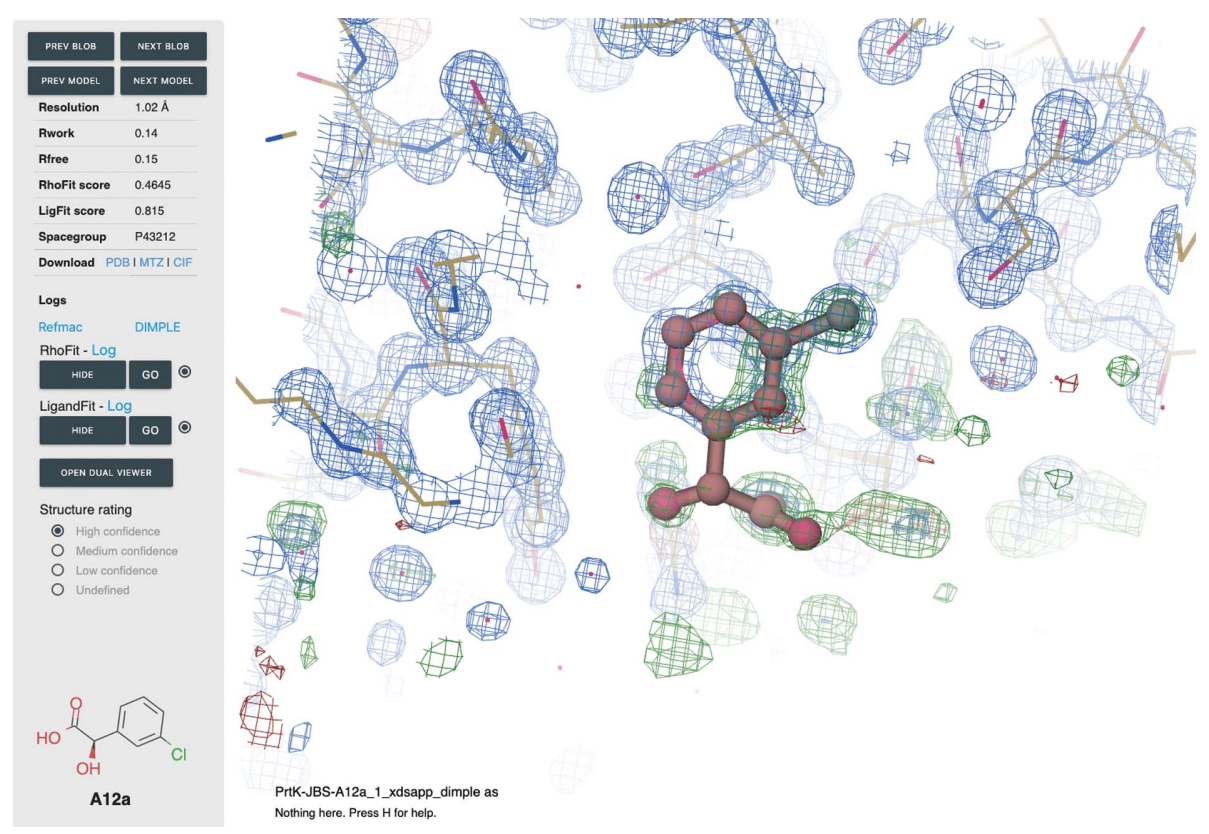

Figure 5

FragMAXapp density viewer. The viewer features unexplained blobs and navigation between the models, information about the result, log access, ligand navigation for automatic ligand-fitting results and structure scores. The density viewer is based on $U g l y M O L$. A full representation of the user interface is available in Supplementary Fig. S5.

\subsection{Export tools}

After the analyses are finished, the web app provides tools to download the data. A variety of choices for which data to export is available, combining process data, log files, structure files and electron densities. Pre-processing results using $P D B$ REDO (Joosten et al., 2012) before deposition is optional but is highly recommended as it improves the quality of automatically refined structures and prepares them for subsequent deposition in the PDB. Using this feature, users are no longer required to manually deposit every model; instead, a compressed archive file with structures, reflection data and the index file is generated, ready to be uploaded using the
OneDep system. The compressed file can be downloaded from FragMAXаpp or using regular tools available at MAX IV. At the time of publication, SFTP and Globus (Foster, 2011) are offered to users. A final option supported by FragMAXapp is uploading of the raw data to public diffraction databases such as proteindiffraction.org (Grabowski et al., 2016).

\section{Fragment screening using FragMAXарp}

To test our implementation of the automated methods, a fragment-screening campaign was performed using proteinase K (PROK) from Tritirachium album (UniProt accession code
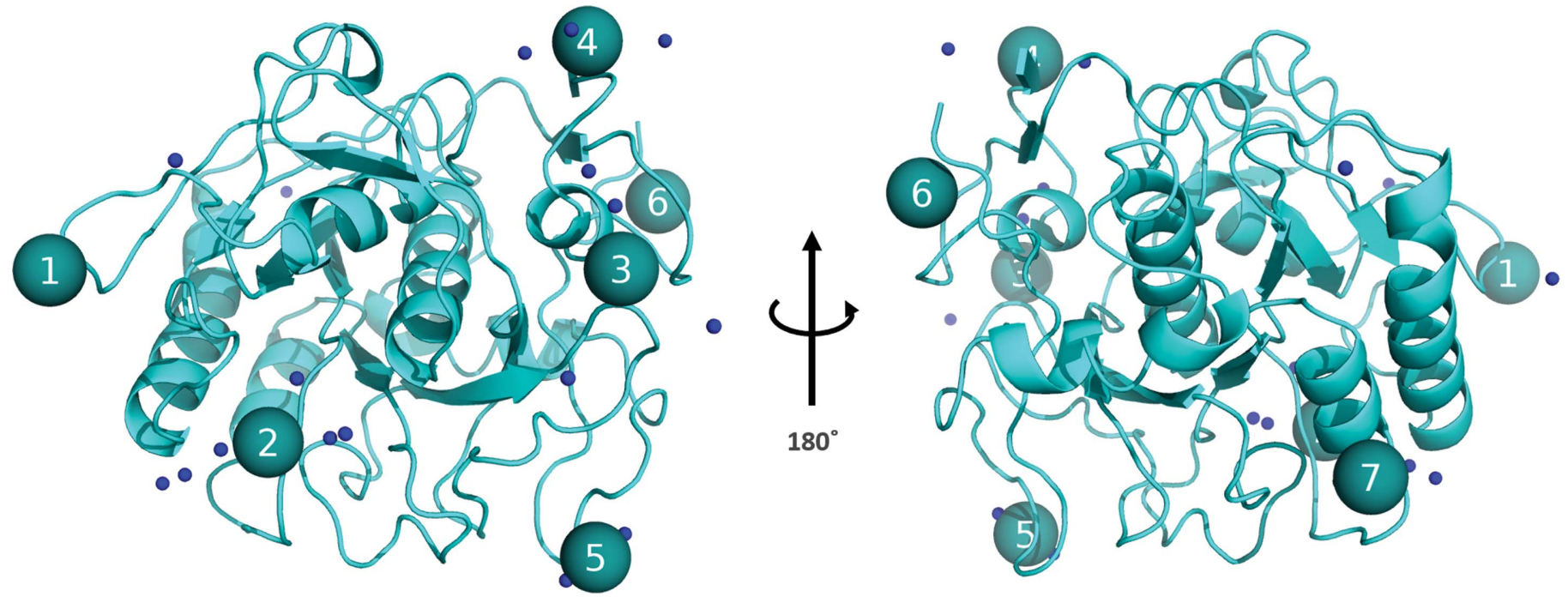

Figure 6

Proteinase K sites numbered from 1 to 7 as identified by PanDDA analysis. 


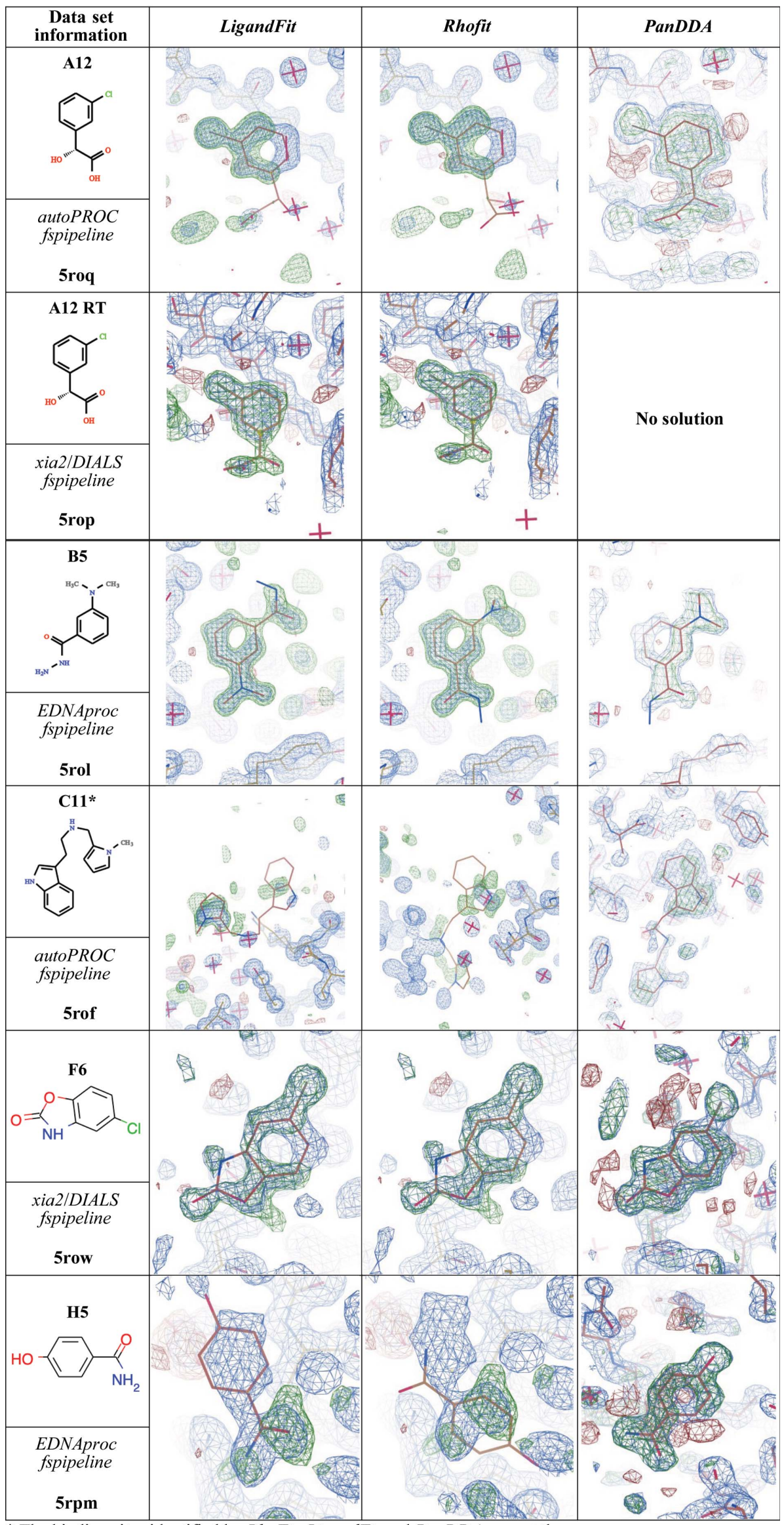

* The binding sites identified by RhoFit, LigandFit and PanDDA are not the same.
P06873). PROK is a study model of serine proteases (Larson et al., 2009). The materials and methods used to obtain PROK crystals, including the soaking and harvesting procedures, are described in Section S1. Data-collection parameters are given in Table 1.

Fragment screening of the 96 samples prepared as ligand soaks using the Frag Xtal Screen library, and an extra 40 apo crystals, was performed and data analysis was performed using FragMAXаpp. The plots, with an overview of data processing and structure refinement, are available in Supplementary Table S1. Applying different automated ligand-fitting (LigandFit and Rhofit) and ligand-searching (PanDDA with manual inspection of $Z$-maps) methods led to very time-efficient hit identification in two weeks of data analysis, including post-refinement and preparation for deposition. This is remarkable when compared with the expected time required to perform the analysis of 136 data sets. At the time of writing this article (GitHub commit https://github. com/FragMAX/FragMAXapp/commit/ 14199af229a43b25a5994c5d58bc92df523cb31d), ligand modelling requires Coot (Emsley et al., 2010), which can be achieved by accessing a virtual computer at synchrotron facility. PanDDA analysis was performed by allowing FragMAXapp to select the best combination of data processing and structure refinement; the selection is available in Section S1. PanDDA identified events and clustered them into seven sites (Fig. 6 ), which were compared with standard difference density map analysis and automated ligand-fitting methods (Fig. 7). In general, when the unexplained density was sufficiently defined to fit a large portion of the corresponding fragment, all methods modelled the bound ligand with a certain degree of precision and would

\section{Figure 7}

Automated ligand-searching comparison. The LigandFit and Rhofit columns take the highest scored ligand from each method. The PanDDA column shows ligands that were manually modelled. Ligands with a different binding site for different methods are annotated. All structures and density maps are publicly available. 
Table 1

Data-collection parameters for PROK crystals at BioMAX.

\begin{tabular}{lll}
\hline & Cryo temperature & Room temperature \\
\hline Wavelength $(\AA)$ & 0.976 & 0.976 \\
No. of images & 3600 & 1800 \\
Oscillation $\left({ }^{\circ}\right)$ & 0.1 & 0.20 (helical scan) \\
Exposure $(\mathrm{ms})$ & 11 & 10 \\
Photon flux (photons s & -1 & $\sim 1 \times 10^{11}$ \\
Beam size $(\mu \mathrm{m})$ & $50 \times 50$ & $50 \times 50$ \\
Resolution $($ at the edge) $(\AA)$ & 1.24 & 1.24 \\
\hline
\end{tabular}

require little real-space refinement to make it ready for deposition. However, using the information from the PanDD $A$ event map is likely to improve the model, as shown for fragments A12 and B5 in Fig. 7. In several cases, such as fragments $\mathrm{B} 7$ and $\mathrm{C} 11$, automated fitting methods disagree on the site in which to locate the ligand, making the $\operatorname{PanDDA}$ event map decisive in ligand modelling. To highlight how each of these methods compares with each other and what the user can typically expect from the automated methods in Frag$M A X a p p$, no further modelling was performed on the protein-ligand complexes. Overall, PanDDA led to an increase in the hit rate, from ten to 18 identified binding events, and a better modelling of bound fragments with partial occupancy.

The results from the PROK fragment screening and the apo structures used to build the ground-state model in PanDDA were submitted to the Protein Data Bank (PDB) using the export tool built into FragMAXapp. Overall, this fragmentscreening campaign is an example of the application of the software and its capabilities, demonstrating what the user can expect from the web app. The fragment-screening platform of MAX IV, including FragMAXapp, has also been used for the validation of a structurally diverse fragment library collection, F2X-Entry, on two protein targets (Wollenhaupt et al., 2020).

\section{Conclusions}

Crystallographic fragment screening is becoming a common method in the early stages of drug-discovery projects, sometimes replacing other biophysical methods as the primary screening technique. Advances in light-source facilities have shifted the bottleneck from generating data to analysing data, requiring equal efforts to improve software support to enable CFS. The strong automation provided at modern MX beamlines, not only for data collection but also for data processing, enables medicinal chemistry-focused groups to utilize structural biology within their research.

FragMAXapp was developed with a focus on providing the necessary tools to manage, process, analyse and export fragment-screening projects from a web browser. The host facility benefits from software that is easy to deploy and develop, based on popular programming languages such as Python and JavaScript. With our plugin-based system, adding or removing functionalities is possible, thus adapting Frag$M A X a p p$ to a variety of scenarios. The user will benefit from a zero-installation setup that is 'ready to go' from any internet- connected device. Furthermore, remote data analysis significantly decreases the time required to obtain results by skipping data transfer and using highly parallelized computing architecture. Similar solutions available at other sites such as XChemExplorer and CRIMS lack the extensive data-analysis features and/or easy access to the application through a web browser. Therefore, FragMAХарp will set the standard for CFS project-management and data-processing tools with its full web-stack implementation, parallel computing support and user-friendly interface.

\section{Availability}

Documentation and source code for FragMAXapp can be obtained from the FragMAX project GitHub page (https:// github.com/FragMAX/FragMAXapp) free of charge for academic use or noncommercial applications. Detailed tutorial and other instructions can be found on the FragMAXapp project page (https://fragmax.github.io/).

\section{Acknowledgements}

The FragMAX group would like to thank all of the BioMAX staff for supporting and improving the beamline that makes the project possible. The authors would like to thank Lionel Trésaugues (Sprint Biosciences AB, Sweden) and Yousif Ali (Linköping University, Sweden) for collaboration during the development of the FragMAXарp encryption mode and for testing the software. The authors would like to thank the PReSTO group for providing the scientific software infrastructure, especially Martin Moche (Karolinska Institutet, Sweden) and Sebastian Thorarensen (Linköping University, Sweden) for promptly providing help when needed. The authors would also like to thank Zdenek Matej (MAX IV Laboratory) for valuable help with the MAX IV computing environment. Finally, the authors would like to thank Nicholas Pearce (Utrecht University, Netherlands) for his insights regarding $P a n D D A$ usage and web implementation. The computations and software access were enabled by resources provided by the Swedish National Infrastructure for Computing (SNIC) at MAX IV, partially funded by the Swedish Research Council.

\section{Funding information}

The following funding is acknowledged: Vetenskapsrådet (grant No. 2018-06454; grant No. 2018-05973); VINNOVA (grant No. 2019-02567). We acknowledge the support by iNEXT-Discovery, project number 871037, funded by the Horizon 2020 program of the European Commission.

\section{References}

Arvai, A. (2012). Adxv - A Program to Display X-ray Diffraction Images. http://www.scripps.edu/ arvai/adxv.html.

Barthel, T., Huschmann, F. U., Wallacher, D., Feiler, C. G., Klebe, G., Weiss, M. S. \& Wollenhaupt, J. (2021). J. Appl. Cryst. 54, 376-382. Berman, H. M., Westbrook, J., Feng, Z., Gilliland, G., Bhat, T. N., Weissig, H., Shindyalov, I. N. \& Bourne, P. E. (2000). Nucleic Acids Res. 28, 235-242. 
Beteva, A., Cipriani, F., Cusack, S., Delageniere, S., Gabadinho, J., Gordon, E. J., Guijarro, M., Hall, D. R., Larsen, S., Launer, L., Lavault, C. B., Leonard, G. A., Mairs, T., McCarthy, A., McCarthy, J., Meyer, J., Mitchell, E., Monaco, S., Nurizzo, D., Pernot, P., Pieritz, R., Ravelli, R. G. B., Rey, V., Shepard, W., Spruce, D., Stuart, D. I., Svensson, O., Theveneau, P., Thibault, X., Turkenburg, J., Walsh, M. \& McSweeney, S. M. (2006). Acta Cryst. D62, 11621169.

Bostock, M. (2017). D3: Bring Data to Life with SVG, Canvas and $H T M L$. https://github.com/d3/d3.

Bricogne, G., Blanc, E., Brandl, M., Flensburg, C., Keller, P., Paciorek, W., Roversi, P., Sharff, A., Smart, O. S., Vonrhein, C. \& Womack, T. O. (2017). BUSTER version 2.10.3. Global Phasing Ltd, Cambridge, UK.

Cipriani, F., Röwer, M., Landret, C., Zander, U., Felisaz, F. \& Márquez, J. A. (2012). Acta Cryst. D68, 1393-1399.

Collaborative Computational Project, Number 4 (1994). Acta Cryst. D50, 760-763.

Davies, T. G. \& Tickle, I. J. (2011). Top. Curr. Chem., 317, 33-59.

Davies, T. G., Van Montfort, R. L. M., Williams, G. \& Jhoti, H. (2006). Fragment-based Approaches in Drug Discovery, edited by W. Jahnke \& D. A. Erlanson, pp. 193-214. Weinheim: Wiley-VCH.

Di Castro, M., Pazos, A., Ridoutt, F., Ristau, U. \& Fiedler, S. (2008). PCaPAC 2008 - International Workshop on Personal Computers and Particle Accelerator Controls, pp. 192-194.

Emsley, P., Lohkamp, B., Scott, W. G. \& Cowtan, K. (2010). Acta Cryst. D66, 486-501.

Evans, P. (2006). Acta Cryst. D62, 72-82.

Foster, I. (2011). IEEE Internet Comput. 15, 70-73.

Fuchs, M. R., Bhogadi, D. K., Jakoncic, J., Myers, S., Sweet, R. M., Berman, L. E., Skinner, J., Idir, M., Chubar, O., McSweeney, S. \& Schneider, D. K. (2016). AIP Conf. Proc. 1741, 030006.

Gildea, R. J., Bourhis, L. J., Dolomanov, O. V., Grosse-Kunstleve, R. W., Puschmann, H., Adams, P. D. \& Howard, J. A. K. (2011). J. Appl. Cryst. 44, 1259-1263.

Grabowski, M., Langner, K. M., Cymborowski, M., Porebski, P. J., Sroka, P., Zheng, H., Cooper, D. R., Zimmerman, M. D., Elsliger, M.-A., Burley, S. K. \& Minor, W. (2016). Acta Cryst. D72, 11811193.

Grimes, J. M., Hall, D. R., Ashton, A. W., Evans, G., Owen, R. L., Wagner, A., McAuley, K. E., von Delft, F., Orville, A. M., Sorensen, T., Walsh, M. A., Ginn, H. M. \& Stuart, D. I. (2018). Acta Cryst. D74, 152-166.

Incardona, M.-F., Bourenkov, G. P., Levik, K., Pieritz, R. A., Popov, A. N. \& Svensson, O. (2009). J. Synchrotron Rad. 16, 872879.

Ito, S., Ueno, G. \& Yamamoto, M. (2019). J. Synchrotron Rad. 26, 1361-1366.

Joosten, R. P., Joosten, K., Cohen, S. X., Vriend, G. \& Perrakis, A. (2011). Bioinformatics, 27, 3392-3398.

Joosten, R. P., Joosten, K., Murshudov, G. N. \& Perrakis, A. (2012). Acta Cryst. D68, 484-496.

Kabsch, W. (2010). Acta Cryst. D66, 125-132.

Krojer, T., Talon, R., Pearce, N., Collins, P., Douangamath, A., Brandao-Neto, J., Dias, A., Marsden, B. \& von Delft, F. (2017). Acta Cryst. D73, 267-278.

Krug, M., Weiss, M. S., Heinemann, U. \& Mueller, U. (2012). J. Appl. Cryst. 45, 568-572.

Landrum, G. (2012). RDKit. The Official Sources for the RDKit Library. https://github.com/rdkit/rdkit.

Larson, S. B., Day, J. S., Nguyen, C., Cudney, R. \& McPherson, A. (2009). Acta Cryst. F65, 192-198.

Long, F., Nicholls, R. A., Emsley, P., Gražulis, S., Merkys, A., Vaitkus, A. \& Murshudov, G. N. (2017). Acta Cryst. D73, 112-122.

Martiel, I., Buntschu, D., Meier, N., Gobbo, A., Panepucci, E., Schneider, R., Heimgartner, P., Müller, D., Bühlmann, K., Birri, M., Kaminski, J. W., Leuenberger, J., Oliéric, V., Glettig, W. \& Wang, M. (2020). J. Synchrotron Rad. 27, 860-863.
McCarthy, A. A., Barrett, R., Beteva, A., Caserotto, H., Dobias, F., Felisaz, F., Giraud, T., Guijarro, M., Janocha, R., Khadrouche, A., Lentini, M., Leonard, G. A., Lopez Marrero, M., Malbet-Monaco, S., McSweeney, S., Nurizzo, D., Papp, G., Rossi, C., Sinoir, J., Sorez, C., Surr, J., Svensson, O., Zander, U., Cipriani, F., Theveneau, P. \& Mueller-Dieckmann, C. (2018). J. Synchrotron Rad. 25, 1249-1260.

Moriarty, N. W., Grosse-Kunstleve, R. W. \& Adams, P. D. (2009). Acta Cryst. D65, 1074-1080.

Mueller, U., Förster, R., Hellmig, M., Huschmann, F. U., Kastner, A., Malecki, P., Pühringer, S., Röwer, M., Sparta, K., Steffien, M., Ühlein, M., Wilk, P. \& Weiss, M. S. (2015). Eur. Phys. J. Plus, 130, 141.

Mueller, U., Thunnissen, M., Nan, J., Eguiraun, M., Bolmsten, F., Milàn-Otero, A., Guijarro, M., Oscarsson, M., de Sanctis, D. \& Leonard, G. (2017). Synchrotron Radiat. News, 30, 22-27.

Murakami, H., Hasegawa, K., Ueno, G., Yagi, N., Yamamoto, M. \& Kumasaka, T. (2020). Acta Cryst. D76, 155-165.

Nascimento, A. F. Z. (2020). 11th International Workshop on X-ray Radiation Damage to Biological Samples.

Noske, G. D., Nakamura, A. M., Gawriljuk, V. O., Fernandes, R. S., Lima, G. M. A., Rosa, H. V. D., Pereira, H. D., Zeri, A. C. M., Nascimento, A. F. Z., Freire, M. C. L. C., Oliva, G. \& Godoy, A. S. (2021). bioRxiv, 2020.12.23.424149.

Nurizzo, D., Bowler, M. W., Caserotto, H., Dobias, F., Giraud, T., Surr, J., Guichard, N., Papp, G., Guijarro, M., Mueller-Dieckmann, C., Flot, D., McSweeney, S., Cipriani, F., Theveneau, P. \& Leonard, G. A. (2016). Acta Cryst. D72, 966-975.

Oscarsson, M., Beteva, A., Flot, D., Gordon, E., Guijarro, M., Leonard, G., McSweeney, S., Monaco, S., Mueller-Dieckmann, C., Nanao, M., Nurizzo, D., Popov, A., von Stetten, D., Svensson, O., Rey-Bakaikoa, V., Chado, I., Chavas, L., Gadea, L., Gourhant, P., Isabet, T., Legrand, P., Savko, M., Sirigu, S., Shepard, W., Thompson, A., Mueller, U., Nan, J., Eguiraun, M., Bolmsten, F., Nardella, A., Milàn-Otero, A., Thunnissen, M., Hellmig, M., Kastner, A., Schmuckermaier, L., Gerlach, M., Feiler, C., Weiss, M. S., Bowler, M. W., Gobbo, A., Papp, G., Sinoir, J., McCarthy, A., Karpics, I., Nikolova, M., Bourenkov, G., Schneider, T., Andreu, J., Cuní, G., Juanhuix, J., Boer, R., Fogh, R., Keller, P., Flensburg, C., Paciorek, W., Vonrhein, C., Bricogne, G. \& de Sanctis, D. (2019). J. Synchrotron Rad. 26, 393-405.

Pearce, N. M., Krojer, T., Bradley, A. R., Collins, P., Nowak, R. P., Talon, R., Marsden, B. D., Kelm, S., Shi, J., Deane, C. M. \& von Delft, F. (2017). Nat. Commun. 8, 15123.

Powell, H. R. (2017). Biosci. Rep. 37, BSR20170227.

Rego, N. \& Koes, D. (2015). Bioinformatics, 31, 1322-1324.

Samara, Y. N., Brennan, H. M., McCarthy, L., Bollard, M. T., Laspina, D., Wlodek, J. M., Campos, S. L., Natarajan, R., Gofron, K., McSweeney, S., Soares, A. S. \& Leroy, L. (2018). Acta Cryst. D74, 986-999.

Sanchez-Weatherby, J., Sandy, J., Mikolajek, H., Lobley, C. M. C., Mazzorana, M., Kelly, J., Preece, G., Littlewood, R. \& Sørensen, T. L.-M. (2019). J. Synchrotron Rad. 26, 291-301.

Schiebel, J., Krimmer, S. G., Röwer, K., Knörlein, A., Wang, X., Park, A. Y., Stieler, M., Ehrmann, F. R., Fu, K., Radeva, N., Krug, M., Huschmann, F. U., Glöckner, S., Weiss, M. S., Mueller, U., Klebe, G. \& Heine, A. (2016). Structure, 24, 1398-1409.

Schurmann, J., Lindhe, I., Janneck, J. W., Lima, G. \& Matej, Z. (2019). 2019 53rd Asilomar Conference on Signals, Systems, and Computers, pp. 978-983. Piscataway: IEEE.

Smart, O. S., Womack, T. O., Flensburg, C., Keller, P., Paciorek, W., Sharff, A., Vonrhein, C. \& Bricogne, G. (2012). Acta Cryst. D68, 368-380.

Smart, O. S., Womack, T. O., Sharff, A., Flensburg, C., Keller, P., Paciorek, W., Vonrhein, C. \& Bricogne, G. (2011). GRADE. Global Phasing Ltd, Cambridge, UK.

Smart, O. S., Womack, T. O., Sharff, A., Flensburg, C., Keller, P., Paciorek, W., Vonrhein, C. \& Bricogne, G. (2014). Rhofit. Global Phasing Ltd, Cambridge, UK. 
Sparta, K. M., Krug, M., Heinemann, U., Mueller, U. \& Weiss, M. S. (2016). J. Appl. Cryst. 49, 1085-1092.

Stetten, D. von, Carpentier, P., Flot, D., Beteva, A., Caserotto, H., Dobias, F., Guijarro, M., Giraud, T., Lentini, M., McSweeney, S., Royant, A., Petitdemange, S., Sinoir, J., Surr, J., Svensson, O., Theveneau, P., Leonard, G. A. \& Mueller-Dieckmann, C. (2020). J. Synchrotron Rad. 27, 844-851.

Svensson, O., Andreu, J., Karpics, I. \& Nan, J. (2020). Lucid3: A Computer Vision Python Library that Detects Crystallography Sample Holders. https://github.com/mxcube/lucid3.

Svensson, O., Gilski, M., Nurizzo, D. \& Bowler, M. W. (2018). Acta Cryst. D74, 433-440.

Terwilliger, T. C., Adams, P. D., Moriarty, N. W. \& Cohn, J. D. (2007). Acta Cryst. D63, 101-107.

Terwilliger, T. C., Klei, H., Adams, P. D., Moriarty, N. W. \& Cohn, J. D. (2006). Acta Cryst. D62, 915-922.

Ursby, T., Åhnberg, K., Appio, R., Aurelius, O., Barczyk, A., Bartalesi, A., Bjelčić, M., Bolmsten, F., Cerenius, Y., Doak, R. B., Eguiraun, M., Eriksson, T., Friel, R. J., Gorgisyan, I., Gross, A., Haghighat, V., Hennies, F., Jagudin, E., Norsk Jensen, B., Jeppsson, T., Kloos, M., Lidon-Simon, J., de Lima, G. M. A., Lizatovic, R., Lundin, M., Milan-Otero, A., Milas, M., Nan, J., Nardella, A., Rosborg, A., Shilova, A., Shoeman, R. L., Siewert, F., Sondhauss, P., Talibov, V. O., Tarawneh, H., Thånell, J., Thunnissen, M., Unge, J., Ward, C., Gonzalez, A. \& Mueller, U. (2020). J. Synchrotron Rad. 27, 1415-1429.

Vonrhein, C., Flensburg, C., Keller, P., Sharff, A., Smart, O., Paciorek,
W., Womack, T. \& Bricogne, G. (2011). Acta Cryst. D67, 293-302. Winn, M. D., Ballard, C. C., Cowtan, K. D., Dodson, E. J., Emsley, P., Evans, P. R., Keegan, R. M., Krissinel, E. B., Leslie, A. G. W., McCoy, A., McNicholas, S. J., Murshudov, G. N., Pannu, N. S., Potterton, E. A., Powell, H. R., Read, R. J., Vagin, A. \& Wilson, K. S. (2011). Acta Cryst. D67, 235-242.

Winter, G. \& McAuley, K. E. (2011). Methods, 55, 81-93.

Winter, G., Waterman, D. G., Parkhurst, J. M., Brewster, A. S., Gildea, R. J., Gerstel, M., Fuentes-Montero, L., Vollmar, M., MichelsClark, T., Young, I. D., Sauter, N. K. \& Evans, G. (2018). Acta Cryst. D74, 85-97.

Wojdyr, M. (2016). UglyMol - View Protein Models and Electron Density. https://uglymol.github.io/.

Wollenhaupt, J., Barthel, T., Lima, G. M. A., Metz, A., Wallacher, D., Jagudin, E., Huschmann, F. U., Hauss, T., Feiler, C. G., Gerlach, M., Hellmig, M., Förster, R., Steffien, M., Heine, A., Klebe, G., Mueller, U. \& Weiss, M. S. (2021). J. Vis. Exp. 3, doi:10.3791/62208.

Wollenhaupt, J., Metz, A., Barthel, T., Lima, G. M. A., Heine, A., Mueller, U., Klebe, G. \& Weiss, M. S. (2020). Structure, 28, 694706.

Wright, N. D., Collins, P., Koekemoer, L., Krojer, T., Talon, R., Nelson, E., Ye, M., Nowak, R., Newman, J., Ng, J. T., Mitrovich, N., Wiggers, H. \& von Delft, F. (2021). Acta Cryst. D77, 62-74.

Yin, X., Scalia, A., Leroy, L., Cuttitta, C. M., Polizzo, G. M., Ericson, D. L., Roessler, C. G., Campos, O., Ma, M. Y., Agarwal, R., Jackimowicz, R., Allaire, M., Orville, A. M., Sweet, R. M. \& Soares, A. S. (2014). Acta Cryst. D70, 1177-1189. 